\title{
Le Parti communiste chinois et le 4 Juin, ou comment s'en sortir et comment s'en débarrasser
}

\section{Michel Bonnin}

\section{(2) OpenEdition}

\section{Journals}

Édition électronique

URL : http://journals.openedition.org/perspectiveschinoises/5215

ISSN : 1996-4609

Éditeur

Centre d'étude français sur la Chine contemporaine

Édition imprimée

Date de publication : 1 juin 2009

Pagination : 58-68

ISSN : 1021-9013

Référence électronique

Michel Bonnin, « Le Parti communiste chinois et le 4 Juin, ou comment s'en sortir et comment s'en débarrasser », Perspectives chinoises [En ligne], 2009/2 | 2009, mis en ligne le 01 juin 2011, consulté le 01 mai 2019. URL : http://journals.openedition.org/perspectiveschinoises/5215

(c) Tous droits réservés 


\title{
Le Parti communiste chinois
}

et le 4 Juin, ou comment

s'en sortir et comment

s'en débarrasser

\author{
MICHEL BONNIN
}

Le mouvement démocratique du printemps 1989 et le massacre du 4 juin ont constitué un très grave défi à la légitimité du Parti communiste chinois. Vingt ans après, il apparâit que le Parti a réussi à le surmonter au prix d'un retour aux fondamentaux politiques de l'Etat-Parti léniniste et d'un recours au nationalisme comme légitimité de remplacement. Mais, malgré tous ses efforts pour occulter et déformer l'histoire de la " tourmente " de 1989 (dont témoignent les manuels scolaires et universitaires), il n’a pas réussi à se débarrasser de cette tache historique. La question de la reconnaissance des responsabilités et celle d'une éventuelle " réconciliation " continuent à se poser.

"La victoire dans la répression des troubles et de la rébellion contre-révolutionnaire [du printemps 1989 à Pékin] a une grandiose signification historique. Elle a protégé les fruits de la victoire de la révolution chinoise, renforcé la position stratégique du socialisme dans notre pays ainsi que les résultats de dix années de réforme et d'ouverture, et elle a fourni au Parti et au peuple une expérience dont ils ont tiré des leçons extrêmement utiles ${ }^{(1)}$."

" J'ai dit à des étrangers que la plus grande erreur que nous avons commise pendant ces dix dernières années a été l'échec de l'éducation. Par là, je voulais dire principalement l'échec de l'éducation politicoidéologique, et pas seulement celle qu'on donne dans les écoles, aux jeunes et aux étudiants ; c'est de l'éducation du peuple tout entier dont je voulais parler." "Dans l'avenir, lorsque nous aurons à régler des problèmes du même genre, il nous faudra tout faire dès leur apparition pour éviter qu'ils ne s'étendent ${ }^{(2)}$. "

$T$ es autorités chinoises affirment qu'elles ont su tirer les leçons des événements du printemps 1989 et que 1 le pouvoir du Parti communiste chinois (PCC) en est sorti renforcé. L'histoire chinoise de ces 20 dernières années confirme, dans une large mesure, cette affirmation.
Le prix de ce succès a été un éloignement durable, pour la Chine, de toute perspective de démocratisation. Dans cet article, nous présenterons d'abord un panorama général des moyens par lesquels le PCC a renforcé son emprise sur le pays, puis nous nous pencherons plus précisément sur le problème que continue à poser le massacre du 4 juin lui-même, sur la façon dont il est " traité », mais non réglé, par le régime et sur les possibilités d'un règlement futur. Si le régime a su se sortir de la crise du 4 Juin, saura-t-il se débarrasser de la cicatrice qu'elle a laissée dans l'histoire contemporaine de la Chine?

\section{Répondre au défi}

Comme le dit l'un des manuels d'histoire que nous présenterons ci-dessous, le mouvement de Tiananmen a été le plus grave défi politique auquel le PCC a été confronté depuis son accession au pouvoir.

La cohésion interne et la légitimité du Parti ont été fortement ébranlées, et cela à une période où le communisme était en plein désarroi en Europe de l'Est et en URSS.

1. M1 (voir note 9 ci-dessous), p. 361.

2. Extraits du discours prononcé par Deng Xiaoping le 9 juin 1989 devant des officiers de l'armée chargée d'appliquer la loi martiale dans la capitale (Deng Xiaoping, CEuvres choisies, tome 3, p.58). 
La déesse de la démocratie érigée sur la place Tian'anmen

(C) AFP

Certes, contrairement à la version officielle, le mouvement lui-même ne remettait pas en cause, au départ, le pouvoir du Parti. Mais, l'attitude intransigeante de la faction dure, soutenue par Deng Xiaoping et d'autres vétérans très puissants, a entrainé une cassure entre le pouvoir et les manifestants, que la répression sanglante a définitivement scellée. Au lendemain du 4 Juin, le Parti était confronté à trois tâches principales : retrouver sa propre cohésion, reprendre le contrôle de la société et notamment celui des esprits, se refaire une légitimité. Son premier réflexe a été de revenir aux méthodes éprouvées de l'État-Parti léniniste, qui avaient été assouplies dans les années 1980.

\section{Le retour aux fondamentaux de l'État-Parti}

L'élément décisif dans ce genre de régime est la cohésion du Parti lui-même, car seules les divisions au sein de la direction peuvent donner l'occasion à des opposants dans la société de s'exprimer avec une certaine ampleur. Cette cohésion a été obtenue avec l'élimination de la faction " réformiste libérale ", c'est-à-dire favorable à une réforme politique accompagnant la réforme économique. Au plus haut niveau, elle a été obtenue par la mise en résidence surveillée de Zhao Ziyang, l'arrestation de son secrétaire Bao Tong, l'éviction de $\mathrm{Hu}$ Qili, proche collaborateur de Zhao et chargé de la propagande au Comité permanent du Bureau politique, de même que celle de Yan Mingfu, chef du Département du Front uni du Comité central, et Rui Xingwen, du secrétariat du Comité central ${ }^{(3)}$. La purge a touché ensuite tous les échelons. De l'autre côté, les conservateurs favorables à une plus grande orthodoxie économique ont été patiemment mais efficacement marginalisés. Un consensus sans précédent s'est donc créé autour de l'idée de la nécessité d'une réforme économique audacieuse accompagnée de la plus grande stabilité politique possible. L'obtention de ce consensus a été largement facilitée par la grande peur suscitée chez de nombreux dirigeants par le mouvement de 1989, puis par celle dont ils ont été frappés au spectacle de l'effondrement du communisme en Europe de l'Est (avec le sort funeste réservé au grand ami de la Chine qu'était Ceaucescu) et à celui de l'éclatement de l'URSS. Les dirigeants ont, en outre, accordé une grande importance à la " construction du Parti ", c'est-à-dire à son fonctionnement régulier, à son développement et surtout à son rôle de direction effective dans tous les domaines d'activité, dogme que Zhao Ziyang avait commencé à assouplir en prônant une séparation nette entre Parti et administra-

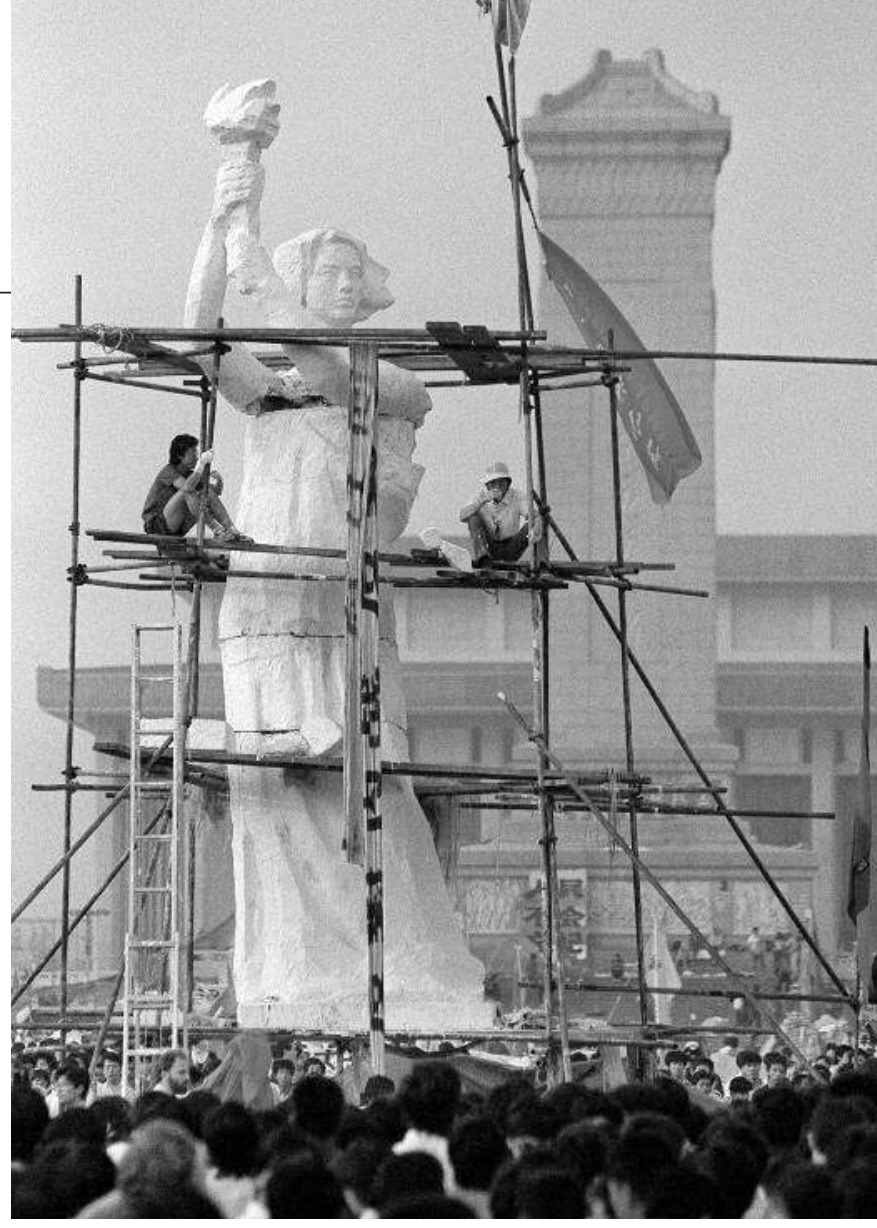

tion. Par ailleurs, le développement à la fois quantitatif et qualitatif du Parti a été assuré par une politique systématique de recrutement au sein des élites économiques et intellectuelles, y compris parmi les étudiants.

La reprise en main de la société s'est faite dans le même esprit systématique. Bien entendu, la première action a consisté à écraser tous les vestiges de la dissidence qui s'était exprimée. Cela s'est fait d'abord par la terreur : le massacre lui-même, bien sûr, mais aussi des semaines pendant lesquelles les tanks paradaient à Pékin, les listes de personnes recherchées étaient diffusées à la télévision et des appels à la délation étaient lancés, les arrestations se multipliaient, parfois suivies d'exécutions rapides et souvent de lourdes condamnations. Après cette période de terreur, les autorités en charge de la sécurité politique, tout en utilisant le système habituel des prisons et des camps de travail dans les cas " graves ", ont mis sur pied des procédures beaucoup plus subtiles et discrètes mais très systématiques de surveillance des personnes "à risque ". Selon la notoriété des individus, il existe depuis lors toute une gradation de mesures allant de la simple surveillance par des policiers en civil à des mesures temporaires de résidence surveillée ou de transfert dans des lieux d'accueil en banlieue, voire dans des stations balnéaires, afin d'empêcher que les dissidents ne puissent faire entendre leur voix à des moments spécifiques auprès de journalistes ou de personnalités de passage.

3. Sur les événements et les acteurs de la crise du printemps 1989, voir J.-P. Béja et al., Le tremblement de terre de Pékin, Paris, Gallimard, 1991 et Zhang Liang, Les archives de Tiananmen, Paris, Éditions du Félin, 2004. 
Parallèlement, toutes les organisations sociales existant hors du Parti et de ses organisations de masse ont été dissoutes après le 4 Juin et contraintes de se réenregistrer auprès des autorités. Cela a permis d'en éliminer un certain nombre, qui s'étaient montrées favorables aux idées du mouvement démocratique ou qui avaient été trop proches de la faction libérale du Parti. Par la suite, le désengagement de l'État de certains secteurs, comme l'éducation et la santé, a conduit les autorités à accepter la création d'un grand nombre de nouvelles organisations, mais des règles strictes ont été édictées pour garantir un regard officiel sur leur fonctionnement et interdire l'apparition d'organisations ayant une large implantation dans tout le pays. Il est évidemment toujours hors de question que le Parti accepte la revendication avancée par les étudiants de 1989 d'un dialogue avec des organisations spontanées représentant différents groupes sociaux.

Un élément essentiel du retour aux fondamentaux a été le renforcement de tout l'appareil en charge de la propagande et du contrôle de la pensée. Deng Xiaoping avait particulièrement insisté, dès son discours du 9 juin cité en exergue ci-dessus, sur la nécessité de remédier à l'échec patent dans ce domaine. Jiang Zemin s'empressait alors d'appeler au renforcement du travail idéologique lors du 4 e plenum du $13^{\mathrm{e}}$ Comité central, le 24 juin 1989. Des moyens et un pouvoir accrus étaient immédiatement dévolus au Département central de la propagande et à tout l'appareil qu'il contrôle ${ }^{(4)}$. Les idées démocratiques " libérales " tenues pour responsables des troubles de 1989 étaient fermement dénoncées et le lien était fait entre ces idées et les efforts réalisés par les pays capitalistes pour susciter une "évolution pacifique " dans les pays socialistes afin de leur faire quitter la voie du socialisme. Depuis cette époque, et malgré l'énorme accroissement du nombre des publications liées au développement de l'économie de marché, les autorités de la propagande ont réussi à conserver un contrôle très serré sur tous les médias et à faire passer dans la population les messages politiques du Parti. Même Internet, malgré le défi nouveau qu'il représente, n'échappe que de façon limitée au contrôle de l'appareil sophistiqué et pléthorique qui a été mis en place à son intention.

Le contrôle des jeunes esprits passe aussi par l'éducation scolaire, et une attention particulière a été donnée à la formation idéologique de la jeunesse depuis le 4 Juin. Nous verrons plus loin comment les manuels d'histoire sont utilisés à cette fin. Les cours d'éducation politique, que tous doivent suivre, sont également le lieu privilégié de l'endoctrinement concernant le marxisme-léninisme et la pensée de Mao Zedong, mais aussi des développements plus récents de l'idéologie officielle. Certes, ce contrôle des esprits n'a que des effets limités, car il est difficile de faire croire aux jeunes d'aujourd'hui qu'ils sont en train de participer à la marche du pays vers la société communiste. Le but est plutôt d'imposer une certaine conformité en leur faisant apprendre ce qu'ils doivent dire dans des circonstances officielles. Mais le Parti a su trouver une autre source, beaucoup plus puissante, de légitimité : le nationalisme.

\section{La construction d'une nouvelle légitimité}

Le nationalisme, présenté sous le terme de patriotisme, a été systématiquement promu dans la population et notamment chez les jeunes, à la suite des événements de 1989. C'est au début des années 1990 que le lever du drapeau est devenu une obligation quotidienne dans les écoles. En 1993, le Département central de la propagande a émis un plan pour le travail de propagande patriotique, prévoyant notamment un accroissement du nombre des "sites d'éducation patriotique " et, en août 1994, le Comité central a promulgué un "Programme national d'éducation patriotique " qui a été le signal d'une grande campagne dans tout le pays. Des fonds ont été attribués pour le développement d'activités diverses et pour la construction de musées et de monuments. L'éducation patriotique est devenue officiellement " la tâche principale dans le travail d'édification de la civilisation spirituelle ». Le nationalisme ainsi développé par les autorités a deux spécificités importantes. D'une part, il insiste sur le fait que la Chine est menacée par les Occidentaux et, d'autre part, il défend systématiquement l'idée que le Parti et la patrie sont indissolubles. Au cours d'une importante réunion sur le travail de propagande, début 1994, Jiang Zemin a affirmé : "La communauté internationale est résolument opposée au fait que la Chine devienne forte et puissante et n'abandonnera jamais ses stratagèmes pour occidentaliser et démanteler la Chine. " Et il ajoutait : " En Chine, l'éducation patriotique, collectiviste et socialiste sont une seule et même chose ; elles se soutiennent mutuellement ${ }^{(5)}$. "C'est aussi à cette époque que les manuels d'histoire et les musées ont systématiquement mis en avant les références à "l'humiliation nationale » subie par la Chine aux $\mathrm{XIX}^{\mathrm{e}}$ et $\mathrm{XX}^{\mathrm{e}}$ siècles, du fait des puissances occidentales ${ }^{(6)}$.

4. Voir A.-M. Brady, Marketing Dictatorship - Propaganda and Thought Work in contemporary China, Rowan and Littlefield Publishers, 2008, p. 44-47.

5. Ibid., p. 49-50.

6. W.A. Callahan, «The Cartography of National Humiliation and the Emergence of China's Geobody », Public Culture, vol. 21 n 1, 2009, p. 141-173. 


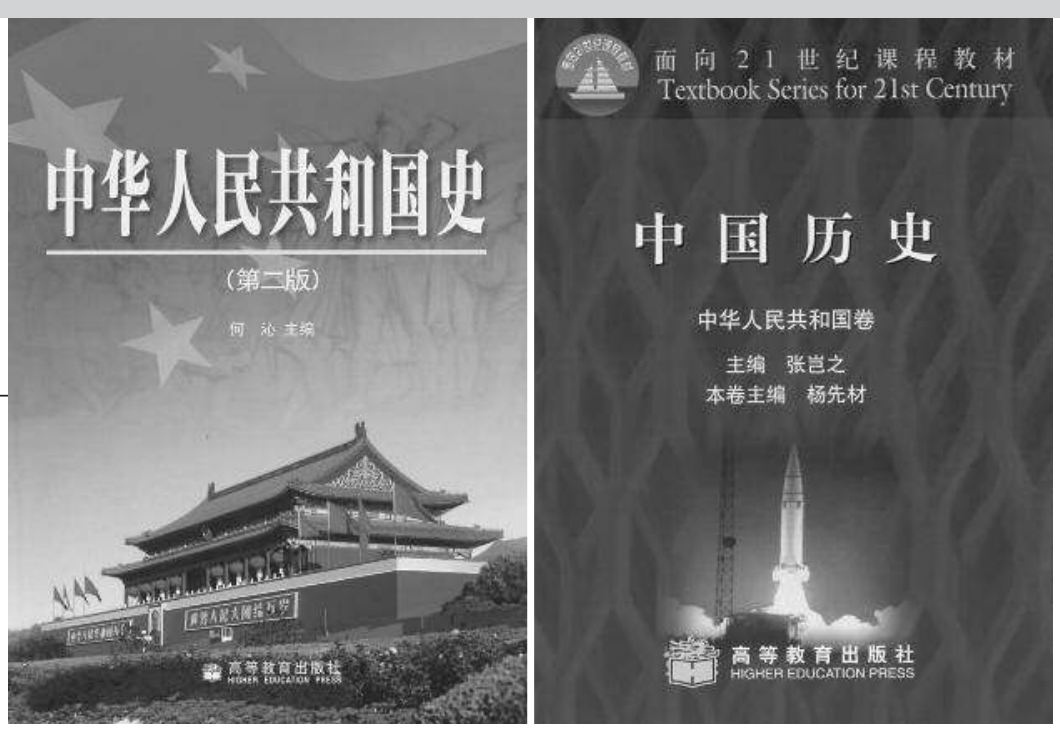

Le recours du pouvoir au nationalisme comme moyen de souder la population autour de lui et de la détourner des idéaux démocratiques d'origine occidentale a été couronné d'un succès certain, notamment dans la jeunesse. Il est vrai que le nationalisme chinois avait une longue tradition, qu'il était relativement facile de revivifier. Ce nationalisme pouvait également s'appuyer sur le sentiment de fierté né des progrès du développement économique et de leurs effets bénéfiques sur le pouvoir d'achat. L'amélioration du niveau de vie de la population était déjà, depuis le début des réformes, la principale source de légitimité du régime, mais elle a connu une accélération à partir des années 1990, grâce à la politique d'insertion de la Chine dans la mondialisation économique. Le pouvoir n'a pas manqué de s'attribuer le mérite de ce succès et de stimuler la fierté nationale, en insistant sur le fait que la Chine était devenue une grande puissance économique et avait vocation à devenir la première puissance au XXI ${ }^{\mathrm{e}}$ siècle.

Mais le renforcement du régime et la capacité qu'il a eue de trouver une nouvelle légitimité après l'épreuve du 4 Juin 1989 ne sont pas suffisants pour faire oublier son " péché originel ". Le spectre du massacre continue à le hanter, comme le montre la fébrilité dont il fait preuve chaque année à l'approche de la date anniversaire, notamment en surveillant tous ceux qui pourraient être tentés de rappeler publiquement ce souvenir, mais aussi en resserrant tous les boulons du contrôle des médias et d'Internet. Ce souvenir, en effet, reste une plaie ouverte dans la société chinoise et l'attitude du Parti face à ce problème est révélatrice de sa puissance, mais aussi de ses faiblesses.

\section{Le * traitement * du 4 Juin par les autorités}

Le contrôle du passé est l'un des éléments de la domination du PCC sur la société chinoise. Il s'applique avec une rigueur particulière concernant les événements sombres de sa propre histoire $^{(7)}$. Sujet particulièrement "sensible ", le mouvement de 1989 est un cas typique, et la façon dont il est traité dans les manuels d'histoire est particulièrement significative.

\section{Le 4 Juin dans les manuels scolaires}

Le premier constat est que la méthode principale est l'occultation complète. C'est celle qui est appliquée dans toute

l'éducation primaire et secondaire. L'idéal est évidemment de faire disparaître purement et simplement cet événement de la mémoire sociale, de le transformer en non-événement pour toute la génération qui ne l'a pas connu directement. Ainsi, les manuels d'histoire pour lycéens ne mentionnent pas le 4 Juin. Le manuel utilisé par le fils de Chen Ziming, à Pékin, saute directement de 1984 à 1998 sur les questions de politique intérieure. En outre, il ne cite pas du tout les noms de dirigeants comme $\mathrm{Hu}$ Yaobang et Zhao Ziyang, qui ont eu pourtant un rôle historique non négligeable mais ne sont pas considérés comme des personnages positifs. Leurs noms n'apparaissant pas non plus dans les médias officiels, ou seulement très rarement, ces dirigeants sont inconnus de la jeune génération ${ }^{(8)}$.

Pour les étudiants d'université dont le curriculum comprend l'histoire de Chine, il était difficile de passer toute cette période sous silence. Mais les manuels à leur disposition donnent une version des événements très orientée. Nous présenterons deux manuels (que nous appellerons Ml et M2), largement utilisés aujourd'hui, tous deux publiés par les Éditions de l'enseignement supérieur ${ }^{(9)}$.

M1 est le dernier tome d'une collection en trois volumes couvrant toute l'histoire de Chine. D'abord publié en 1997, il a été mis à jour pour une seconde édition parue à l'occasion du $50^{\mathrm{e}}$ anniversaire de la fondation de la République populaire de Chine, en 1999. M2 est le sixième et dernier

7. M. Bonnin, «L'histoire de la Révolution culturelle et la mémoire de la "génération perdue" sont-elles condamnées à l'oubli ? », Perspectives chinoises, n 4, 2007, p. 54-66. Voir aussi d'autres contributions dans le même numéro.

8. Meiguo zhi yin, 7/4/2009, repris à l'adresse : http://www.secretchina.com/news/ 287503.html

9. Manuel 1 (M1) : He Qin (éd.), Zhonghua renmin gongheguo shi, di er ban (Histoire de la République populaire de Chine, 2e édition), Gaodeng jiaoyu chubanshe (Higher Education Press), septembre 1999 (1ère édition avril 1997), 494 p. (l'exemplaire consulté a été acheté en janvier 2009 à Shanghai. II s'agit de la 16e impression, datée de décembre 2007). Manuel 2 (M2) : Yang Xiancai (éd.), Zhongguo lishi - Zhonghua renmin gongheguo juan (Histoire de Chine - La République populaire de Chine), Gaodeng jiaoyu chubanshe (Higher Education Press), juillet 2001 (1ère édition), 356 p. (l'exemplaire consulté a été acheté en janvier 2009 à Shanghai. II s'agit de la 10e impression, datée mars 2008). Fait partie de la collection Mianxiang 21 shiji kecheng jiaocai (Textbook Series for 21st Century). He Qin est professeur au département d'histoire du Parti de I'Université du peuple et Yang Xiancai chercheur au Centre de recherches sur l'histoire du Parti dépendant du Comité central. 
volume d'une collection couvrant également toute l'histoire de Chine. Il est paru en 2001 dans la série des "manuels pour le $\mathrm{XXI}^{e}$ siècle». Le travail pour la collection en six volumes a commencé en 1994. Chaque phrase a donc été longuement pesée et discutée. Le public visé comprend les étudiants de licence qui ont l'histoire de Chine au programme, c'est-à-dire les étudiants d'histoire, de chinois, de philosophie et de sciences sociales (il est précisé qu'ils n'ont pas besoin de tout lire, mais seulement de choisir des passages), ainsi que les étudiants de maitrise et de doctorat dans ces matières, de même que les enseignants d'histoire (M2, p. 2). Le manuel $\mathrm{M} 1$ précise clairement dans son introduction les objectifs visés par la publication d'une histoire de la République populaire de Chine (RPC) (MI, p. 2-3) :

$1^{\circ}$ ) « Renforcer l'esprit patriotique et la cohésion nationale. "Tout en se félicitant que l'époque de la faiblesse de la Chine soit dépassée et que peuple chinois puisse enfin " relever la tête " comme jamais dans son histoire, les auteurs ajoutent : « Mais il faut aussi reconnaitre que notre pays n'est pas encore assez puissant [...], et qu'il y aura encore toutes sortes de difficultés et de risques, notamment du fait que la frénésie des forces hostiles étrangères ne s'éteint pas, que l'hégémonisme continue à se développer, et que ces forces ne cessent de guetter l'occasion de fomenter des troubles, dans l'espoir insensé de renverser la Chine socialiste. "Il faut donc continuer à renforcer l'héritage patriotique et la cohésion nationale "pour que la Chine socialiste ne soit jamais dans la position du perdant et continue d'avancer victorieusement ».

$2^{\circ}$ ) "Raffermir la foi socialiste. "Après avoir affirmé que c'est le Parti communiste chinois armé du marxisme-léninisme et de la pensée de Mao Zedong qui a mené la Chine à l'indépendance nationale et au développement, les auteurs notent avec fierté : "Aujourd'hui, à un moment où la cause socialiste a subi de graves revers dans le monde, en Chine, le socialisme continue à faire preuve de vitalité et d'énergie. " Selon eux, malgré les erreurs commises dans le passé sur la voie du socialisme, le PCC a su trouver une théorie, la théorie du "socialisme aux couleurs de la Chine ", qui a montré qu'elle était appropriée à la situation chinoise. Cela a permis de "renforcer la foi dans la victoire finale du socialisme". C'est sur la base de cette foi que l'on doit " unir le peuple pour obtenir, dans le siècle nouveau, de nouvelles victoires dans l'édification d'un puissant État socialiste moderne ".

$3^{\circ}$ ) «Étudier et maitriser les théories concernant le bon gouvernement du pays. » " L'histoire montre " que la pensée de Mao Zedong était la théorie appropriée à la phase révolutionnaire et d'installation du pouvoir et que la théorie de
Deng Xiaoping l'était pour la phase suivante de développement. Ainsi, « l'étude de l'histoire de la RPC revient à étudier et maitriser la pensée de Mao Zedong et la théorie de Deng Xiaoping ». Les auteurs précisent que dans la Chine d'aujourd'hui, c'est cette dernière théorie qui joue le rôle principal et que la maitrise de cette théorie est « la plus sûre garantie pour vaincre toutes les difficultés sur notre route, pour rejeter toutes les interférences néfastes et pour surmonter toutes les épreuves. "

L'objectif d'endoctrinement par l'histoire est donc clairement affiché, l'essentiel étant que tout ce qui est écrit dans le manuel soit " correct " (zhengque), c'est-à-dire politiquement correct ${ }^{(10)}$. Ainsi, le chapitre X, qui conclut l'ouvrage, est intitulé : "Levons haut le grandiose drapeau de la théorie de Deng Xiaoping pour entrer victorieusement dans le nouveau siècle ", slogan lancé par Jiang Zemin pour la célébration du cinquantenaire de la RPC. La fin est consacrée aux raisons pour lesquelles les Chinois peuvent avoir confiance dans l'avenir : $1^{\circ}$ ) le fait que le Parti et l'État leur aient assigné " des objectifs de lutte clairs "; $2^{\circ}$ ) l'expérience acquise depuis la fondation du régime et notamment depuis la politique de réforme et d'ouverture $; 3^{\circ}$ ) la situation de stabilité sociale et politique ; et $4^{\circ}$ ) une " immense énergie spirituelle " fondée sur l'esprit patriotique et la cohésion nationale. Les auteurs notent avec satisfaction dans la dernière page du livre "la marée patriotique sans précédent " qui a suivi le bombardement par un avion de l'OTAN de l'Ambassade de Chine à Belgrade, quelques mois avant la sortie de leur ouvrage. Ils se réjouissent également que " cet acte barbare perpétré par l'OTAN avec à sa tête les États-Unis [ait] amené les gens à réfléchir profondément, à découvrir le vrai visage de l'hégémonisme et de la politique de superpuissance des États-Unis et à reconnaitre leur nature impérialiste, à déceler le caractère hypocrite de la démocratie à l'américaine et l'ambition stratégique des États-Unis consistant à contenir la Chine et à exercer une hégémonie mondiale. "

Ainsi, dans la Chine d'aujourd'hui, un manuel d'histoire est aussi un manuel de combat, destiné à " armer " (wuzhuang) la jeunesse de la pensée correcte pour qu'elle puisse participer efficacement aux " objectifs de lutte clairs » qui lui sont assignés. On ne sera donc pas surpris par les omissions et distorsions de faits qui entachent la partie concernant le mouvement démocratique du printemps 1989, ni de leur interprétation tendancieuse, ni du ton militant employé.

10. L'éditeur utilise trois fois ce qualificatif pour décrire le manuel dans les sept lignes de présentation au début du livre. 
Le manuel M2 est plus court, donc moins détaillé, mais il est mieux structuré dans son argumentation, plus clair et plus concis dans le style, légèrement plus modéré aussi dans le ton et les idées. La différence entre les deux se lit sur la couverture : celle de $\mathrm{M} 1$ représente sur toute la page la fameuse tribune de Tiananmen avec le portrait de Mao au centre, le tout sur fond de drapeau rouge aux cinq étoiles, alors que celle de M2 présente une photo de taille réduite du lancement de la fusée spatiale chinoise sur un fond de figures géométriques d'un rouge foncé beaucoup moins agressif que celui de M1. Il s'agit là, cependant, de différences de détail. Pour l'essentiel, les deux manuels donnent la même présentation de l'histoire, souvent avec les mêmes mots, les mêmes arguments, les mêmes citations... et les mêmes oublis. Cette uniformité dans la différence tient au fait qu'il existe une seule version officielle de l'histoire. Pour l'épisode qui nous intéresse ici, c'est celle qui a été mise au point dans les déclarations officielles qui ont suivi le 4 Juin et surtout celle qui a été présentée dans le discours de Deng Xiaoping du 9 juin, puis développée à partir de ce texte fondateur au cours du $4^{e}$ plenum du $13^{e}$ Comité central, les 23 et 24 juin 1989. Dans M1, le passage s'intitule " La tourmente politique (zhengzhi fengbo) de 1989, la convocation du 4 e plenum du $13^{e}$ Comité central ». Il prend environ huit pages, sans divisions internes (p. 358-366).

Dans M2, il s'appelle « La répression de la tourmente politique de 1989 " et couvre un peu plus de trois pages (p. 309-312). Il est divisé en deux parties : « La tourmente politique de 1989 et sa répression " et "L'établissement d'un nouveau collectif dirigeant central au 4 e plenum du $13^{\mathrm{e}}$ Comité central ».

Les deux textes commencent par analyser les causes de la tourmente (fengbo):

Dans M1, la responsabilité est attribuée d'abord à Zhao Ziyang. On lui reproche d'avoir prématurément interrompu le mouvement contre la libéralisation bourgeoise dès le 15 mars 1987, attaqué les marxistes au sein du Parti, critiqué le travail idéologique et politique et de ne pas avoir lutté contre le développement de la corruption. Par ailleurs, l'inflation et les désordres économiques ont entrainé un mécontentement dans la population, qui a mal compris (wujie) ce qui se passait. Cette situation a été utilisée par les partisans du libéralisme bourgeois pour attaquer la direction du Parti, prôner l'occidentalisation complète et le capitalisme. Une force réactionnaire s'est peu à peu créé à travers des réunions et des pétitions demandant un changement de régime politique. Les forces hostiles étrangères ont alors renforcé leur stratégie d'évolution pacifique en exerçant des pressions. Le déferlement de la pensée "sociale-démocrate " qui a touché à l'époque certains pays socialistes d'Europe de l'Est a encouragé encore les éléments libéraux-bourgeois. C'est ce qui explique les troubles (dongluan) qui se sont déroulés dans le pays du 15 avril au 4 juin et la rébellion contre-révolutionnaire (fangeming baoluan) qui a eu lieu à Pékin.

Dans $\mathrm{M} 2$, les idées sont à peu près les mêmes, mais l'argumentation est mieux structurée et le style plus clair et concis. L'événement est décrit comme une " tourmente politique grave " (yanzhong de zhengzhi fengbo), dont l'ampleur et la violence étaient sans précédent depuis 1949. On affirme qu'il a constitué un test très sévère pour le Parti et pour sa politique définie au $3^{e}$ plenum du $11^{e}$ Comité central. On ajoute que les causes en sont multiples et, dès le début du texte, on introduit une certaine distanciation en reproduisant l'appréciation postérieure de Deng Xiaoping, selon lequel : "Cette tourmente devait arriver à un moment ou à un autre. Elle a été déterminée par le macroclimat international et le microclimat de la Chine. Elle devait venir. Ce n'est pas quelque chose qui pouvait être changé par la volonté humaine. " (Aujourd'hui comme à l'époque, cette idée permet de décharger Deng lui-même et le PCC en général d'une lourde responsabilité.) Les auteurs commencent par parler des causes extérieures en insistant sur les efforts produits par le " monde impérialiste occidental " pour faire abandonner la voie socialiste aux pays socialistes, sur le renforcement du recours à l' "évolution pacifique " et sur le déferlement des idées sociales-démocrates en URSS et dans les pays de l'Europe de l'Est. À l'intérieur, on reproche à certains dirigeants de tous niveaux, y compris au niveau central, d'avoir négligé le travail idéologique. (Ici, la différence avec M1 est sensible : le nom de Zhao Ziyang n'est même pas cité.) On reproche à une infime minorité (ji shaoshu) des cadres dirigeants d'avoir profité de leur pouvoir pour obtenir des avantages personnels, ce qui a provoqué un grave problème de corruption entrainant un affaiblissement du prestige du Parti. L'inflation a par ailleurs réduit le niveau de vie qui n'avait fait que croître depuis les réformes, entraînant mécontentement et incompréhension dans la population. Tous ces phénomènes ont encouragé des fanatiques de la libéralisation bourgeoise à demander le pluralisme idéologique, l'occidentalisation totale et la privatisation, aggravant ainsi le trouble dans les esprits.

Sur les événements eux-mêmes, les différences entre les deux manuels sont minimes :

Selon M1 : le déclencheur a été le décès de Hu Yaobang, le 15 avril. « Une infime minorité de partisans du libéralisme bourgeois ont pensé que c'était une bonne occasion. Ils ont alors fomenté des troubles politiques qui fermentaient depuis 
longtemps et dont l'objectif était le renversement de la direction du le Parti communiste chinois, le renversement de la Chine socialiste. »L'hommage à Hu était justifié, mais l'infime minorité, sous prétexte de lui rendre hommage, a lancé des rumeurs mensongères selon lesquelles la mort de $\mathrm{Hu}$ était le résultat d'une persécution politique, si bien que les activités commémoratives des étudiants se sont rapidement transformées en manifestations de type politique. D'un coup, les rumeurs se sont répandues et des attaques contre les principaux dirigeants du Parti et de l'État, contre la direction par le Parti communiste et contre le système socialiste sont apparues sur des affiches, des banderoles et dans des slogans. En quelques jours, on a assisté à des actions illégales comme l'incident grave de l'attaque de Xinhuamen ${ }^{(I I)}$, l'occupation de la place Tiananmen et des manifestations de grande ampleur. "Ils ont aussi créé des organisations illégales comme la Fédération autonome des étudiants ", et ils (le contenu de ce « ils » n'est pas précisé) ont excité les masses, si bien que les manifestations se sont étendues à tout le pays. En outre, à Xi'an, Changsha et Chengdu, on a assisté à des émeutes et autres activités criminelles. Les fauteurs de troubles (dongluan zhizaozhemen) ont utilisé le mouvement étudiant pour soulever une série de revendications politiques constituant leur programme, dont les deux plus importantes étaient : 1) réévaluer l'action de $\mathrm{Hu}$ Yaobang et rejeter la façon dont le Comité central lui avait demandé de démissionner en 1987 ; 2) rejeter la lutte contre la libéralisation bourgeoise et réhabiliter les personnes qui en avaient été les cibles. Ainsi, dès le début, les troubles ont mis en avant l'antagonisme aigu entre la libéralisation bourgeoise et les Quatre principes fondamentaux ${ }^{(12)}$. Mais, face à ces troubles, Zhao Ziyang a adopté une attitude de tolérance et en fait de soutien.

Le 23 avril, Zhao Ziyang est parti en Corée du Nord. Le 24, Li Peng a convoqué une réunion du Comité permanent du Bureau politique, qui a décidé après examen des faits que l'on se trouvait d'ores et déjà face à des troubles politiques anti-Parti et anti-socialistes planifiés et organisés. Il a été décidé de "mobiliser sans réserve les masses, de chercher à rassembler la majorité et à isoler la minorité et de lutter avec force pour mettre rapidement fin aux troubles ». Le 25, Deng Xiaoping a prononcé un discours important dans lequel il apportait son plein soutien aux décisions du Comité permanent. Le 26 avril, le Quotidien du peuple publiait un éditorial reprenant ces idées et appelant tout le monde à "lutter pour mettre rapidement fin à ces troubles ".

Après la parution de l'éditorial, grâce au renforcement de l'éducation politique et idéologique apportée aux étudiants par les organisations du Parti à tous les niveaux, de nombreux étudiants ont compris la nature de ce combat. La situation des établissements d'enseignement supérieur de Pékin et des autres villes a commencé à se stabiliser. À ce moment-là, Zhao Ziyang revenu depuis quelques jours au pays, a brusquement changé son attitude positive à l'égard du discours de Deng Xiaoping, affirmant que la caractérisation du mouvement contenue dans l'éditorial du 26 avril était erronée et devait être corrigée.

Ce passage est intéressant : il commence par un énorme mensonge historique concernant l'effet de l'éditorial. On ne dit pas un mot sur la grande manifestation du 27 avril qui est le véritable baptême du mouvement, puisqu'en décidant de poursuivre leurs activités malgré la condamnation et les menaces contenues dans l'éditorial du Quotidien du peuple, les étudiants lançaient à Deng Xiaoping et à Li Peng un défi sans précédent dans l'histoire de la RPC. Non contents d'occulter cet événement essentiel, les auteurs affirment exactement le contraire de la réalité quand ils écrivent que la situation s'est stabilisée grâce à l'éditorial relayé par les organisations du Parti. Et ils continuent logiquement dans leur construction d'une explication entièrement fondée sur le complot d'une poignée de libéraux bourgeois d'un côté et la trahison de Zhao Ziyang de l'autre, en présentant les actes qui sont reprochés à Zhao. Ils parlent de son discours du 4 mai à la Banque asiatique de développement qui allait à l'encontre des décisions du Parti, qui montrait au grand jour les divisions au sein de la direction et encourageait les fauteurs de troubles. Zhao est accusé d'avoir fait, le 6 mai, un discours qui encourageait la presse à se libéraliser, le résultat étant " l'apparition dans l'opinion d'une tendance erronée à soutenir le mouvement étudiant et les troubles ». Les organisateurs de ces troubles ont alors joué deux cartes : 1) affirmer qu'ils souhaitaient un « dialogue » avec le gouvernement (là aussi, le mensonge est grossier, puisque la revendication des étudiants de dialoguer avec le gouvernement est présente dès le début du mouvement $\left.{ }^{(13)}\right) ; 2$ ) organiser une grève de la faim : "Le 13 mai, à l'instigation d'une infime minorité, une partie des étudiants ont fait la grève de la faim sur la

11. Les 19 et 20 avril, dans la soirée, des étudiants ont organisé un sit-in devant Xinhuamen, la porte Sud de Zhongnanhai, résidence des plus hauts dirigeants du pays proche de Tiananmen. C'est là qu'ont eu lieu les premières échauffourées avec la police armée.

12. Voie socialiste, dictature démocratique du peuple, direction par le Parti communiste, marxisme-léninisme-pensée-maozedong.

13. Voir J.-P. Béja et al., Le tremblement de terre de Pékin, op. cit., p. 157. 
place Tiananmen. " Cette action a entrainé une sympathie dans la population, si bien que des centaines de milliers de personnes ont exprimé leur soutien. L'importante réunion au sommet sino-soviétique (le nom honni de Gorbatchev n'est pas prononcé) a été en partie gâchée par ces activités. Le 16 au soir, une réunion d'urgence du Comité permanent a été convoquée, mais n'a pas pris de décision à cause du refus de Zhao. Le 17, le nombre de manifestants et les attaques contre Deng Xiaoping ont atteint des sommets sans précédent. Le 17 au soir, une réunion du Comité permanent a décidé l'établissement de la loi martiale dans la mesure où la police ne pouvait faire face. Une réunion des cadres de la capitale était convoquée pour le 19 au soir, afin de prendre des décisions radicales pour mettre fin aux troubles, mais Zhao Ziyang a refusé d'y participer, montrant quil rompait ouvertement avec le Parti. En accord avec la Constitution, la loi martiale était programmée avec effet le 20 à 10 heures du matin. Mais les planificateurs des troubles ont continué à occuper la place, à créer des incidents et à aggraver la situation, si bien que les troubles se sont transformés à Pékin en rébellion contre-révolutionnaire. (Rien n'est dit sur le fait que c'est la population pékinoise qui s'est massivement opposée à l'entrée des troupes dans la ville. Une "infime minorité » en aurait été bien incapable.) Le 3 juin, alors qu'une partie des troupes de la loi martiale entrait pour s'installer à leur poste, les chefs des organisations illégales ont placé des barricades à certains carrefours, ont bloqué les camions militaires, et il y a même eu des camions incendiés et des officiers et soldats tués. La fin du passage mérite d'être reproduite intégralement : "Ils ont même planifié d'utiliser le fait que le lendemain était un dimanche pour rameuter plus de monde dans les rues, créant une situation d'émeute, dans l'intention de renverser d'un coup le gouvernement et de prendre le pouvoir. Dans cette situation extrêmement critique, le 3 juin au soir, le Comité central du Parti, le gouvernement et la commission militaire centrale n'ont pas eu d'autre choix que de prendre une décision déterminée et d'ordonner aux troupes de la loi martiale stationnées aux alentours de Pékin d'entrer par la force et d'étouffer la rébellion contre-révolutionnaire. Dans leur progression, confrontées à une situation intolérable, les troupes ont été contraintes de se défendre par les armes contre les attaques barbares des émeutiers. Le 4 juin à l'aube, les quelques milliers d'étudiants restés sur la place Tiananmen ont été convaincus et sommés de quitter les lieux pacifiquement par la troupe, qui a fait preuve d'une patience et d'une persévérance extrêmes. "

La version présentée ici est contredite par tous les témoignages et les documents consultables sur cet événement, y compris ceux des nombreux médias occidentaux dont les correspondants étaient sur place. Elle est écrite sur un ton très agressif et est particulièrement virulente contre Zhao Ziyang. On a le sentiment que les auteurs sont restés très proches des discours de l'époque, notamment de ceux du dirigeant le plus en pointe dans l'hostilité aux étudiants, le Premier ministre Li Peng.

En conclusion de ce passage, les auteurs donnent la citation de Deng Xiaoping sur le caractère inévitable de cet événement qui, dans M2, a été placée en introduction aux événements.

Dans M2, la présentation est pratiquement identique, mais avec moins de détails. La dénonciation de Zhao Ziyang est un peu moins virulente, mais les critiques qui lui sont adressées sont les mêmes.

\section{Les effets du contrôle idéologique de la jeunesse}

On ne peut aujourd'hui que constater l'efficacité relativement grande de l'occultation du mouvement de Tiananmen. L'immense majorité des jeunes d'aujourd'hui, même en ville, ne sait pas qu'il y a eu un massacre perpétré par l'Armée populaire de libération en plein Pékin à cette époque. Et alors que l'image du jeune homme en chemise blanche qui, le 5 juin 1989, a eu le courage de se tenir debout devant une colonne de chars est connue dans le monde entier, le réalisateur du documentaire The Tankman, sorti en 2006, montre que de jeunes étudiants pékinois d'aujourd'hui ne connaissent pas cette image et sont même totalement incapables de deviner à quoi elle correspond ${ }^{(14)}$. Quelques-uns de mes amis pékinois m'ont fait part de l'ébahissement manifesté par leur enfant quand ils se sont décidés à lui révéler ce qui s'était passé dans la nuit du 3 au 4 juin 1989. Ces jeunes gens, qui étaient déjà étudiants, étaient sidérés d'apprendre qu'un tel événement avait eu lieu dans leur ville, alors qu'ils étaient déjà nés, sans quills en aient jamais entendu parler. Seuls, certains enfants d'activistes du mouvement démocratique ou de familles de victimes ont entendu parler du 4 Juin par leurs parents dès leur jeune âge. Bien entendu, l'efficacité de cette omerta officielle ne tient pas qu'au contrôle des manuels scolaires, mais aussi à celui de l'ensemble des médias, y compris Internet, sur lequel sont pourchassées systématiquement toutes les références à l'événement. Et parmi ceux qui ont entendu

14. Ce film peut être vu à l'adresse : http://www.pbs.org/wgbh/pages/frontline/ tankman/view/

.


parler de la " tourmente politique " de 1989 au cours de leurs études ou lors d'activités politiques officielles, nombreux sont ceux qui croient à la version officielle d'un trouble contre-révolutionnaire manipulé par les forces anti-chinoises de l'étranger.

\section{Le spectre du 4 Juin}

Les autorités chinoises maintiennent donc totalement l'interprétation des événements qu'elles ont donnée à l'époque et n'ont jamais exprimé le moindre regret concernant les pertes humaines qui restent impossibles à chiffrer du fait de l'interdiction absolue de tout débat ou de toute enquête sur le sujet. Cependant, malgré tous les efforts faits pour imposer l'oubli de cet événement, efforts qui ont une efficacité réelle dans la jeune génération, le 4 Juin 1989 continue à hanter la société. Non seulement, les familles qui ont perdu un enfant, les participants qui ont souffert dans leur chair et ceux, très nombreux, qui ont été moralement traumatisés ou du moins bouleversés et révoltés par ce massacre ne pourront jamais l'oublier. Mais, même pour ceux qui ne l'ont pas connu, qui l'ignorent ou qui n'en connaissent que la version officielle, la possibilité reste qu' un jour ils apprennent la vérité. L'existence de ce cadavre caché dans un placard commande donc irrémédiablement le comportement de ceux qui se savent coupables et qui, en conséquence, déploient toute leur astuce et toute leur énergie pour que le placard ne soit jamais ouvert.

Dans ces conditions, cette plaie ne peut évidemment pas se refermer chez les victimes, mais, au-delà de cette injustice faite à plusieurs milliers de familles, le 4 Juin constitue un nœud gordien figeant les relations entre le pouvoir et la population dans une situation malsaine faite de mensonge d'un côté et de défiance de l'autre. Tout relâchement dans le contrôle des publications et des médias, et notamment dans le monopole de l'interprétation de l'histoire, porte le risque d'un dérapage qui conduirait le pouvoir à devoir répondre à des questions portant sur la réalité de ce qui s'est passé en 1989 et, finalement, sur sa responsabilité dans cette affaire. Le 4 Juin joue donc un rôle aggravant dans le blocage de la réforme politique en Chine.

Comment sortir de cette situation bloquée ? C'est une question à laquelle ont réfléchi beaucoup d'intellectuels chinois, notamment parmi les participants et les observateurs directs de l'époque. L'écrivaine Dai Qing a fait sensation lorsqu'elle a publié en mars 2009, dans la revue de Hong Kong Yazhou Zhoukan, un article appelant à la réconciliation entre les autorités et la population à propos du 4 Juin, sur le modèle sud-africain de la Commission vérité et réconciliation ${ }^{(15)}$. Selon elle, pour parvenir à la réconciliation, il faut d'abord établir la vérité et il faut que les deux côtés, gouvernement et étudiants, reconnaissent leurs erreurs.

L'idée de la réconciliation nécessaire n'a pas été inventée par Dai Qing. Depuis mars 2007, il existe, par exemple, un Think-tank de la réconciliation chinoise (Zhongguo hejie zhiku) regroupant des intellectuels chinois vivant en Chine ou à l'étranger, et dont le responsable principal est un universitaire chinois vivant en Australie. Ce groupe a commencé à mettre sur pied une "Commission d'enquête vérité et réconciliation ». Des participants connus du mouvement de 1989 comme Chen Ziming, Liu Xiaobo et Zhou Duo en font partie et ont défendu publiquement l'idée de réconciliation $^{(16)}$. On la trouve également dans la Charte 08 (point 19 du chapitre intitulé "Nos propositions "), ce qui n'est pas étonnant puisque de nombreux membres du "think-tank " sont des signataires de la Charte ${ }^{(17)}$. Mais, si la majorité des membres du mouvement démocratique déclarent souhaiter un jour une réconciliation, la plupart d'entre eux notent que cela ne peut se faire que sur la base d'une reconnaissance des faits et des responsabilités de la part des autorités. La Charte 08 est particulièrement claire sur ce point. Or, comme le fait remarquer Wang Dan, il n'existe aucun signe montrant que le pouvoir souhaite s'orienter dans cette direction, mais au contraire un renforcement du contrôle sur ce sujet sensible ${ }^{(18)}$.

Tant que les mêmes personnes, ou leurs héritiers politiques directs, sont au pouvoir, le principal obstacle à une telle entreprise de vérité historique consiste évidemment dans la crainte des responsables de devoir rendre des comptes. Certes, on pourrait imaginer que, comme dans le cas de l'Afrique du Sud, les participants à une telle commission

15. Cette commission, créée en 1993, était présidée par Mgr Desmond Tutu, archevêque du Cap et prix Nobel de la paix. Son objet était la recension et l'amnistie éventuelle des crimes et exactions politiques commis au nom du gouvernement sud-africain mais également au nom des mouvements de libération nationale, depuis 1960. Elle a contribue à la réconciliation entre les deux communautés, noire et blanche, après la fin de la politique d'apartheid.

16. Voir " Aozhou Zhongguo hejie zhiku » (Le "Think-tank de la réconciliation chinoise" d'Australie), Yazhou Zhoukan, 15 mars 2009. Voir les objectifs et la liste des membres http://zxwh.cc333.com/cgibin/index.dIl?page6? webid=cc333\&userid=2242535\&columnno=10\&articleid=934

17. Pour une traduction française de la Charte 08 , voir par exemple, la traduction publiée sur le site de la revue Rue 89 du 17 décembre 2008. Concernant la mise sur pied d'une commission d'enquête Vérité, la charte ne limite pas son champ au 4 Juin, mais l'étend à toutes « les injustices et atrocités passées ».

18. Yawei, «Zhongguo minjian renshi tichu liusi shehui da hejie » (Une personnalité non officielle chinoise propose une grande réconciliation à propos du 4 Juin), consulté à l'adresse : http://www.voanews.com/chinese/archive/2009-03/w2009-03-18-voa66.cfm?CFID $=173048944 \&$ CFTOKEN $=87543325 \&$ jsessionid $=003081$ b60956f0aad4442e $52445 f 661$ e4264 
acceptent dès le départ le principe du pardon individuel pour les responsables. Mais, même si cela était accepté par les victimes, les autorités ne pourraient tolérer une telle commission dans l'état actuel des rapports entre pouvoir et société. Comme le fait remarquer le philosophe Zhang Boshu, de l'Académie des sciences sociales de Chine :

La réconciliation est emblématique d'une certaine culture politique. Il s'agit d'un lien qui s'établit sur un pied d'égalité, d'un échange, d'un compromis. Cette tradition manque réellement en Chine.

S'il y a une " tragédie chinoise ", c'est sans doute celle de cette incapacité du pouvoir à dialoguer avec la société. C'est ce refus de quitter un tant soit peu sa position de supériorité radicale qui a empêché le PCC de répondre sincèrement à la demande de dialogue formulée par les étudiants, et qui a mené Deng Xiaoping, dès le 25 avril, à dénoncer contre toute vraisemblance le mouvement spontané des étudiants comme "des troubles organisés par des conspirateurs et visant essentiellement à rejeter la direction des affaires par le Parti communiste et le système socialiste ". C'est cette conception du pouvoir comme une citadelle à défendre qui l'a conduit ensuite à décider d'utiliser la violence la plus brutale contre la prétendue "rébellion contre-révolutionnaire ". Le drame est que la société semble désarmée pour faire sortir le pouvoir de son enfermement dans une arrogance paranoïaque. Les débats qui ont eu lieu sur Internet à propos de différentes prises de position de membres du Think-tank pour la réconciliation chinoise ont souligné la difficulté de la tâche. En effet, si le PCC n'a pas l'intention de reconnaitre ses torts, il ne se laissera pas prendre à des manifestations de bonne volonté destinées à le contraindre à faire ce qu'il ne veut pas faire. Il demandera toujours plus de renonciations, ce qui coupera les participants à cet éventuel dialogue de leur propre base. La critique principale des internautes, visant notamment les paroles de Dai Qing, est qu'en demandant que chaque côté reconnaisse ses erreurs, on met sur le même pied les erreurs tactiques et organisationnelles des étudiants d'une part et les crimes dont s'est rendu coupable le régime d'autre part. D'où la demande inquiète d'un partisan de la réconciliation : " Je souhaite que l'élite intellectuelle chinoise qui prône la réconciliation ne défende pas par la même occasion une mentalité de peuple servile ${ }^{(19)}$. "

Plusieurs commentateurs ont montré qu'il existait des traits de la réconciliation sud-africaine (conflit entre races différentes, rôle positif de la religion et du cardinal Tutu, etc.) qui n'étaient pas présents dans le cas du 4 Juin. L'événement sans doute le plus comparable serait plutôt le massacre de Kwangju en Corée du Sud en $1980^{(20)}$. Mais, plus fondamentalement, si le cas sud-africain est devenu un modèle, c'est parce qu'il constituait un brillant symbole d'un processus de transition démocratique pacifique. Or, le Parti communiste chinois n'a cessé de répéter, et particulièrement ces derniers mois, que la Chine ne pouvait en aucun cas suivre le modèle politique « occidental ${ }^{(21)}$ ». Ainsi, malgré le sincère désir de réconciliation de Dai Qing et de beaucoup d'autres intellectuels chinois, ils doivent bien reconnaitre qu' « il faut être deux pour danser le tango » et que, tant que le pouvoir ne se sentira en sécurité qu'en position de supériorité absolue, il sera très difficile de combler le fossé qui sépare la forteresse du pouvoir des manants qu'il dirige.

\section{Le 4 Juin et l'avenir du régime}

Les 20 années qui ont suivi le 4 Juin 1989 ont donc vu la conjonction d'un bond en avant économique et d'un bond en arrière politique. $\mathrm{Ce}$ grand écart a produit un régime énigmatique, un objet politique non identifié, fascinant par sa vitalité et sa souplesse dans le domaine socio-économique, mais assez inquiétant par sa rigidité politique intérieure et extérieure. On peut estimer, comme l'intellectuel du mouvement démocratique $\mathrm{X}_{\mathrm{u}} \mathrm{Ben}$, que le 4 Juin a fait passer le régime du PCC de la période post-totalitaire des années 1980 à une phase néo-totalitaire, plus dure et surtout plus éloignée d'une éventuelle transition démocratique ${ }^{(22)}$. Il est vrai que le qualificatif d'" autoritaire " pour caractériser la forme de contrôle que nous avons décrite ci-dessus parait bien faible. Si un régime autoritaire pouvait fort bien pratiquer un massacre comme celui du 4 juin, l'occultation systématique de cet événement au point qu'il soit inconnu par toute la jeune génération est un exploit qui renvoie à un type tout à fait spécifique de régime.

Mais, quoi qu'il en soit du choix des concepts les plus appropriés, il faut souligner qu'il existe, malgré tout, une continuité dans l'ensemble de la période de la politique de « réfor-

19. Duli pinglun (The Independent Review), 3 novembre 2008 : www.duping.net/XHC/show. php?bbs=11\&post $=909435$

20. Voir, par exemple, Mo Li, « Liusi wenti jiejue fangshi zhi tantao » (Discussion sur les moyens de résoudre le problème du 4 Juin), Zhengming, $n^{\circ} 378$, avril 2009, p. 77-79.

21. Voir, par exemple, le discours de Jia Qinglin à la réunion de l'Assemblée politique consultative du peuple (Agence Chine nouvelle, 18 janvier 2009), le rapport présenté par Wu Bangguo devant la session annuelle de l'Assemblée nationale populaire (Agence Chine nouvelle, 9 mars 2009), ainsi que les articles consacrés à ce sujet par plusieurs intellectuels officiels, page 7 du Quotidien du peuple du 9 février 2009.

22. Xu Ben, "Zhongguo de "xin jiquanzhuyi" ji qi moshi jingxiang " (Le "néo-totalitarisme" chinois et son spectacle de fin d'un monde), Dangdai Zhongguo yanjiu, vol. $12, n^{\circ} 4$, 2005, p. 4-26. 
me et ouverture " (gaige kaifang) et que celle-ci est liée à la personnalité et à la volonté d'un homme : Deng Xiaoping. Deng est l'homme du développement sous la dictature, et son poids a été décisif, tant à la fin des années 1970 qu'à la fin des années 1980. L'acte fondateur de sa politique est double : d'une part, la politique de gaige kaifang, victorieuse au fameux 3 e plenum du $11^{\text {e }}$ Comité central, fin 1978, qui fait de Deng le véritable numéro un et, d'autre part, son discours du 30 mars 1979, qui fixe les limites politiques de la réforme en instituant les Quatre principes fondamentaux et qui signale le début de l'écrasement du premier Printemps de Pékin ${ }^{(23)}$. Il est vrai que Deng n'est qu' un parmi d'autres dans la mise au point de la réforme. Des dirigeants comme Hu Yaobang, Wan Li ou Zhao Ziyang, y prennent une part importante. Mais, c'est Deng qui fixe l'orientation générale avec sa politique d'équilibre entre gaige kaifang et respect des Quatre principes fondamentaux résumée dans le slogan " saisir avec les deux mains " (liang shou zhua). Et, grâce au fameux " facteur ginseng " (qui a permis à de nombreux vétérans du PCC d'être encore en état d'intervenir en 1989), Deng est encore assez vigoureux, dix ans plus tard, pour réitérer cet acte fondateur double, mais dans le sens inverse cette fois : d'abord la répression du 4 juin, qui est l'affirmation sanglante des Quatre principes fondamentaux et, ensuite, le voyage dans le Sud du début de l'année 1992, qui est le lancement d'une nouvelle étape de la politique de réforme et d'ouverture dans le domaine économique.

$\mathrm{Ce}$ que le 4 Juin a effectivement changé, et qui peut justifier l'idée qu'on est passé dans une phase nouvelle, c'est que la crise a convaincu l'ensemble des dirigeants de s'en tenir strictement au système de l'État-Parti léniniste et d'abandonner la perspective d'une réforme politique profonde. Le fait que les deux secrétaires généraux du Parti qui se sont succé- dé depuis le 4 Juin, Jiang Zemin et Hu Jintao, n'aient pas dévié de la politique définie par Deng Xiaoping montre que celle-ci répondait aux intérêts de la couche dirigeante et qu'elle était viable. Il n'empêche que les coûts énormes de cette politique en ce qui concerne la liberté individuelle et notamment la liberté de penser et de créer, le respect des droits de l'homme, la lutte contre la corruption, l'harmonie et l'équité sociales, ainsi que la participation des citoyens à la vie politique empêchent de penser que cette politique répond aux intérêts à long terme du pays tout entier. Il est certainement malsain pour un régime politique de ne pas oser regarder son histoire en face et d'imposer à son peuple un oubli forcé ou une mémoire distordue. Comme à la fin des années 1970, seule une courageuse réévaluation des verdicts passés, et notamment de ceux qui concernent les revendications démocratiques de 1989, permettra à la Chine de trouver le chemin d'un développement plus harmonieux. •

$\begin{array}{ll}\text { Glossaire } & \\ \text { feizhengfu zuzhi } & \text { 非政府組織 } \\ \text { dongluan } & \text { 動亂 } \\ \text { dongluan zhizaozhemen } & \text { 動亂製造者們 } \\ \text { fangeming baoluan } & \text { 反革命暴亂 } \\ \text { fengbo } & \text { 風波 } \\ \text { gaige kaifang } & \text { 改革開放 } \\ \text { ji shaoshu } & \text { 極少數 } \\ \text { liang shou zhua } & \text { 雨手抓 } \\ \text { wujie } & \text { 誤解 } \\ \text { wuzhuang } & \text { 武装 } \\ \text { yanzhong de zhengzhi fengbo } & \text { 嚴重的政治風波 } \\ \text { zhengque } & \text { 正確 } \\ \text { zhengzhi fengbo } & \text { 政治風波 } \\ \text { Zhongguo hejie zhiku } & \text { 中國和解智庫 }\end{array}$

